\title{
Empiric antibiotics therapy for mildly elevated prostate specific antigen: Helpful to avoid unnecessary biopsies?
}

\author{
Andrea Fandella ${ }^{1}$, Sara Benvenuto ${ }^{1}$, Elisa Guidoni ${ }^{1}$, Marco Giampaoli ${ }^{2}$, Alessandro Bertaccini ${ }^{2}$ \\ ${ }^{1}$ Urology, Casa di Cura Giovanni XXIII Monastier, Treviso, Italy; \\ ${ }^{2}$ Clinica Urologica, Alma Mater Studiorum, Università di Bologna, Italy.
}

\begin{abstract}
Summary Purpose: The management of mildly elevated (4.0-10.0 $\mathrm{ng} / \mathrm{ml})$ prostate specific antigen (PSA) is uncertain. Immediate prostate biopsy, antibiotic treatment, or monitoring PSA level for 1-3 months is still in controversy.

Materials and Methods: We retrospectively analysed the effect of empiric antibiotics on an increased PSA in a mono-institutional study. We analysed the data of 100 patients with a PSA of 4-10 ng/ml and normal digital rectal examination undergoing their first prostate biopsy. Patients were divided in two different cohorts. One cohort was submitted to antibiotic therapy (Levoxacin $500 \mathrm{mg}$ daily for 20 days) and both cohort had a re-dosing of PSA before the prostate biopsy.

Results: Average age of the whole group of patients was $66.48 \pm 8.32$ years and their average initial PSA level was $6.67 \pm 1.57 \mathrm{ng} / \mathrm{mL}$. In the treated group $(\mathrm{N}=49) 29$ patients had a decreasing PSA value from mean baseline PSA value of $6.6 \pm 1.54 \mathrm{ng} / \mathrm{ml}$ to the re-dosed mean PSA level of $5.4 \pm 1,61 \mathrm{ng} / \mathrm{ml}(p=0.7) ; 20$ patients didn't experience a decrease PSA value, with a mean PSA level of $6.9 \pm 1.68 \mathrm{ng} / \mathrm{ml}$. In the control group $(\mathrm{N}=51), 30$ patients had a decrease of PSA level from mean baseline PSA level of $6.5 \pm 1,59 \mathrm{ng} / \mathrm{ml}$ to a re-dosed PSA level of $5.5 \pm 1.57 \mathrm{ng} / \mathrm{ml}$; 21 patients didn't experience a decrease of PSA value, with a mean PSA level of $6.7 \pm 1.71 \mathrm{ng} / \mathrm{ml}$. Multivariate analysis of age, PSA changes, antibiotics therapy and biopsy results (presence or absence of cancer) revealed no significant difference between the two cohorts. Sepsis after biopsy occurred in 3 patient in the antibiotics group (6\%) and in one of the control group (2\%).

Conclusions: The study, even with some limitations, does not seem to show an advantage due to the administration of antibacterial therapy to reduce PSA values before prostate biopsy and subsequently to reduce unnecessary prostate biopsies.
\end{abstract}

KEY WORDS: PSA; Antibiotics; biopsy; Prostate cancer; Fluorquinolone.

Submitted 21 August 2014; Accepted 30 August 2014

\begin{abstract}
INTRODUCTION
Prostate specific antigen (PSA) is a serum protein secreted by prostate tissue both in benign and malignant conditions. Elevated values of serum PSA are not pathognomonic for prostate cancer but they can be found in various clinical conditions, including inflammation and infection (1).

In men with an increasing PSA without clinical evidence of infection, a common clinical approach is to empirically prescribe antibiotics and subsequently re-dose the PSA.

Until today, several researchers have examined the impact of empiric antibiotics therapy in patients with an increased PSA, in order to find a balanced costs/effective therapy to avoid unnecessary prostate biopsies and to decrease patient discomfort and morbidity from biopsies $(2,3)$.

The common rational is to treat a subclinical prostate infection, having as result a lower serum PSA. This might lead to lower the rate of unnecessary prostate biopsies, since unproven sub clinical prostatitis are responsible for the majority of false positive PSA elevations (4-6).

However, most of the studies available in the literature are limited because they lack a control arm, so it is unclear if the observed PSA changes were secondary to natural variations or to the effect of the antibacterial therapies (7).

The potential disadvantages of an empiric antibiotic approach include unnecessary expenses, side effects and possible adverse reactions related to the drug intake and an increase in multidrug resistant organisms (8).

Our goal was to investigate if an antibiotic therapy in patients eligible for prostate biopsies might be effective in order to avoid unnecessary biopsies so reducing false positive PSA elevation.
\end{abstract}

\section{Materials AND Methods}

We conducted a retrospective mono-institutional study (Casa di Cura Giovanni XXIII Monastier, Treviso, Italy).

We analysed the data of two different cohort of patients, all submitted to their first prostate biopsy set in the last 3 years in our institution. The first cohort $(N=49)$ included patients treated with an antibiotic therapy (Levofloxacin $500 \mathrm{mg}$ daily) for at least 20 days and then 
submitted to PSA re-dosing prior to the prostate biopsy. Otherwise, the second cohort $(\mathrm{N}=51)$ included patients with no antibiotic therapy but with PSA re-dosing before prostate biopsy.

Both cohort presented PSA levels between 4.0-10 ng/mL, no prior diagnosis of prostate cancer or pre-neoplastic lesions (HGPIN/ASAP), a negative digital rectal examination (DRE) and no clinical or laboratory signs of urinary infections (negative urine sediment and negative urine culture).

The analysed data included patient's age and history. Cases with reported events that could have falsely elevated the PSA result (e.g. urinary tract infection, urinary retention, urinary catheterization...) were excluded from the study. All the specimens were analysed in our laboratory (using Beckam Coulter access II Immunoassay System PSA). Analysis of data [age, PSA changes, antibiotic therapy, biopsy result (presence of cancer)] was carried out using SPSS statistical analysis software (IBM SPSS Statistics 21). Data of the second cohort $(N=51)$ was used as control group. Kolmogorov-Smirnov test (cut off at $\mathrm{p}<0.01$ ) was performed. Continuous variables were described using mean \pm standard deviation. Categorical variables were described using frequency distributions (frequency \%). PSA values prior to and following antibiotic therapy were compared using the Student's-t-test for independent samples and a multivariate logistic regression analysis was performed. All tests were two-sided and considered significant at $\mathrm{p}<0.05$. All the patients underwent to a 12-core prostate biopsy according to our standard biopsy procedure (Presti procedure) (9).

\section{RESULTS}

Average age of the whole group of patients (first and second cohort) was $66.48 \pm 8.32$ years and average initial PSA level was $6.67 \pm 1.57 \mathrm{ng} / \mathrm{mL}$ with no significant difference between first and second cohort.

In the treated cohort $(\mathrm{N}=49) 29$ patients showed a decrease of PSA values from mean baseline PSA of $6.6 \pm$ $1,54 \mathrm{ng} / \mathrm{ml}$ to a mean re-dosed PSA of $5.4 \pm 1,61 \mathrm{ng} / \mathrm{ml}$ $(\mathrm{p}=0.7)$, while 20 patients did not experience a lowering of PSA value, with a mean PSA level of $6.9 \pm 1.68$ $\mathrm{ng} / \mathrm{ml}$. In the sub-group with decreasing PSA levels $(\mathrm{N}=$ $29,59 \%$ of first cohort) 6 patients turned out to have prostate cancer (21\%), 5 chronic inflammation (17\%) and 18 benign prostatic hyperplasia (BPH) (62\%).

In the sub-group with no decrease of PSA levels $(\mathrm{N}=20,41 \%$ of first cohort 4 patients demonstrated to have prostate cancer (20\%), 8 chronic inflammation (40\%) and $8 \mathrm{BPH}(40 \%)$. In the control group $(\mathrm{N}=51), 30$ patients showed a decrease of PSA values from a mean baseline PSA of $6.5 \pm 1,59 \mathrm{ng} / \mathrm{ml}$ to a mean redosed PSA of $5.5 \pm 1,57 \mathrm{ng} / \mathrm{ml}$, while 21 patients did not experience a lowering of PSA value, with a mean PSA level of $6.7 \pm 1.71$ $\mathrm{ng} / \mathrm{ml}$.

In the sub-group with decreasing PSA level ( $\mathrm{N}$ $=30,59 \%$ of second cohort) 7 patients turned out to have prostate cancer (23\%), 5 chronic inflammation $(17 \%)$ and $18 \mathrm{BPH}(60 \%)$.

In the sub-group with no decrease of PSA levels $(\mathrm{N}=21$, $41 \%$ of second cohort) 6 patients demonstrated to have prostate cancer (29\%), 3 chronic inflammation (14\%) and $12 \mathrm{BPH}(57 \%)$.

The difference in PSA changes between the two cohort was not statistically significant $(\mathrm{p}=0.3)$. Furthermore there weren't any statistically significant differences between the two sub-groups in each cohort for cancer detection ( $p>0.5)$

Multivariate analysis of age, PSA changes, antibiotics therapy and biopsy results (presence or absence of cancer) revealed no significant difference between the two cohorts ( $\mathrm{P}$ value $>0.05$ in all categories).

Table 1 shows the distribution of diagnoses and performance of biopsies for each subgroup. Prostate cancer detection rates were not significantly associated with the changes in PSA (either decreasing or increasing).

Sepsis after biopsy occurred in 3 patient in the antibiotics group ( $6 \%$ ) and in 1 of control group (2\%).

\section{Discussion}

In chronic prostatitis, it has been shown that total PSA and free PSA are all significantly higher in patients with infection. A course of fluoroquinolone therapy in patients with chronic bacterial prostatitis resulted in a median PSA decrease from 8.3 to $5.3 \mathrm{ng} / \mathrm{ml}$ (10).

On the other hand, Habermacher et al. (11) demonstrated that most cases of asymptomatic prostatitis are not caused by bacteria, thus eliminating the rationale for antibacterial therapy.

In the study of Kaygisiz et al. (5), antibiotics were administered to 48 patients who underwent to prostate biopsies. The PSA levels decreased below $4 \mathrm{ng} / \mathrm{mL}$ in 18 (37\%) of them and the biopsies of these men were negative for malignancies. In the subgroup of other 30 men prostate cancer was found in $10.8 \%$. The Authors suggested a long course of antibiotic treatment (at least 3 weeks), regardless of inflammation findings, when PSA levels are mildly high (i.e. $4-10 \mathrm{ng} / \mathrm{mL}$ ), in order to decide whether or not to carry out the biopsy on the basis of the subsequent re-dosed PSA results.

Bozeman et al. reported that when serum PSA had been normalized with treatment there was no longer an indication for transrectal ultrasound-guided biopsy in almost
Table 1.

\begin{tabular}{|c|c|c|c|c|}
\hline \multirow[b]{2}{*}{ Significant decrease of PSA (> 10\%) } & \multicolumn{2}{|c|}{$\begin{array}{l}\text { Cohort } 1 \\
49 \text { pts } \\
\text { Antibiotics }\end{array}$} & \multicolumn{2}{|c|}{$\begin{array}{l}\text { Cohort } 2 \\
51 \text { pts } \\
\text { No antibiotics }\end{array}$} \\
\hline & +29 & -20 & +30 & -21 \\
\hline Prostate cancer & 6 & 4 & 7 & 6 \\
\hline Chronic inflammation & 5 & 8 & 5 & 3 \\
\hline BPH only & 18 & 8 & 18 & 12 \\
\hline $\begin{array}{l}\text { + affirmative/- negative } \\
\text { BPH = prostate benign hyperplasia } \\
\text { PSA = prostate specific antigen } \\
\text { pts = patients }\end{array}$ & & & & \\
\hline
\end{tabular}


half of their 95 patients diagnosed with elevated PSA and chronic inflammation, suggesting that chronic prostatitis is an important cause of elevated PSA and that, when identified, treatment can decrease the percent of negative biopsies (12). A recent editorial by Scardino criticized the unjustified use of antibiotics in a group of patients similar to ours. He emphasized the various inherent disadvantages associated with this approach, such as costs, toxicity, and the promotion of resistant bacterial species development that would have exposed the biopsied patient to more resistant and aggressive sepsis (8).

Significant fluctuations in PSA should raise the suspicion of inflammation or infection as an aetiology, however in these patients it remains controversial; empiric antibiotics therapy prior to a prostate biopsy in order to "normalize" the PSA value remains a non evidence-based practice. Those who underwent a course of fluoroquinolone antibiotics treatment should not have a prostate biopsy within one month from completing the therapy to allow the colonic flora to re-establish itself to a normal state. Akduman et al. demonstrated that patients who received 3 weeks of fluoroquinolones before biopsy had a significantly greater incidence of post-biopsy sepsis $(5.4 \%$ vs $1.7 \%$ ) and all sepsis episodes were attributable to quinolone resistant bacteria (13). Other studies have similarly shown that previous therapy with quinolones predisposes to rectal flora resistance (14).

The results of this study seems to show no advantages due to an empiric antibiotic therapy (full dose floroquinolone for 20 days, in this specific case) to reduce PSA values and avoid unnecessary biopsy in patients with PSA levels between $4-10 \mathrm{ng} / \mathrm{mL}$ and no signs or symptoms of infections. A PSA decrease after antibiotic therapy does not rule out prostate cancer and at the same time a lack of decrease does not exclude it. Thus antibiotics therapy does not seem to eliminate unnecessary prostate biopsy.

Our trial does have some limitations: patients number and retrospective design. We had few cases with histological evidence for chronic prostatitis in our study. This might explain why administration of antibacterial therapy was not helpful in our series.

We also studied only a single class of antibiotic for 20 days, which we believed to be the most commonly used in current clinical practice. It is possible that a different class or length of therapy might alter the above findings. It is possible that a larger prospective trial might identify a clinical benefit for empiric antibiotic treatments.

\section{Conclusions}

It is of crucial importance to use properly and responsibly antibiotics. In patients with an increased PSA, antibiotics are appropriate if there is any clinical suspicion or evidence of urinary infection. Any rise of PSA should be an indication to repeat PSA testing. At the same time we advocate the use of antibiotic therapies only if a bacterial cause has been identified. Empiric use doesn't seem to be of clinical benefit in absence of a clinical or laboratory evidence of infection and it might paradoxically be harmful. Repeating a new PSA test before scheduling a biopsy remains the only acceptable approach.

\section{REFERENCES}

1. Ornstein DK, Smith DS, Rao GS, et al. Biological variation of total, free and percent free serum prostate specific antigen levels in screening volunteers. J Urol. 1997; 157:2179-82.

2. Lorente JA, Arango O, Bielsa O, et al. Effect of antibiotic treatment on serum PSA and percent free PSA levels in patients with biochemical criteria for prostate biopsy and previous lower urinary tract infections. Int J Biol Markers. 2002; 17:84-9.

3. Ozen H, Aygün C, Ergen A, et al. Combined use of prostate-specific antigen derivatives decreases the number of unnecessary biopsies to detect prostate cancer. Am J Clin Oncol. 2001; 24:610-3.

4. Kaygisiz O, Ugurlu O, Kosan M, et al. Effects of antibacterial therapy on PSA change in the presence and absence of prostatic inflammation in patients with PSA levels between 4 and $10 \mathrm{ng} / \mathrm{mL}$. Prostate Cancer Prostatic Dis. 2006; 9:235-8.

5. Baltaci S, Suer E, Haliloglu AH, et al. Effectiveness of antibiotics given to asymptomatic men for an increased prostate specific antigen. J Urol. 2009; 181:128.

6. Serretta V, Catanese A, Daricello G, et al. PSA reduction (after antibiotics) permits to avoid or postpone prostate biopsy in selected patients. Prostate Cancer Prostatic Dis. 2008; 11:1485.

7. Erol H, Beder N, Caliskan T, et al. Can the effect of antibiotherapy and anti-inflammatory therapy on serum PSA levels discriminate between benign and malign prostatic pathologies? Urol Int. 2006; 76:20-6.

8. Scardino PT. The responsible use of antibiotics for an elevated PSA level. Nat Clin Pract Urol. 2007; 4:1.

9. Presti JC jr; Prostate biopsy strategies. Nat Clin Pract Urol. 2007; 4:505-11.

10. Schatteman PH, Hoekx L, Wyndaele JJ, et al. Inflammation in prostate biopsies of men without prostatic malignancy or clinical prostatitis: correlation with total serum PSA and PSA density. Eur Urol. 2000; 37:404-12.

11. Habermacher GM, Chason JT, Schaeffer AJ. Prostatitis/chronic pelvic pain syndrome. Ann Rev Med. 2006; 57:195-206.

12. Bozeman CB, Carver BS, Eastham JA, Venable DD. Treatment of chronic prostatitis lowers serum prostate specific antigen. J Urol. 2002; 167:1723-6.

13. Akduman B, Akduman D, Tokgoz H, et al. Long-term fluoroquinolone use before the prostate biopsy may increase the risk of sepsis caused by resistant microorganisms. Urology 2011; 78:250.

14. Owens RC Jr., Ambrose PG. Antimicrobial safety: focus on fluoroquinolones. Clin Infect Dis. 2005 (suppl.); 41:S144.

\section{Correspondence}

Andrea Fandella, MD

afandella@libero.it

Sara Benvenuto, MD

Elisa Guidoni, MD

Casa di Cura Giovanni XXIII Monastier, Treviso, Italy

Marco Giampaoli, MD

giampaoli.marco85@gmail.com

Alessandro Bertaccini, MD (Corresponding Author)

alessandro.bertaccini@gmail.com

Clinica Urologica, Alma Mater Studiorum, Università di Bologna

Ospedale Sant'Orsola-Malpighi, Bologna, Italy 